\section{Igualdade de oportunidade entre os estados brasileiros: \\ uma análise microeconométrica com \\ base nos dados da PNAD 2009}

\section{Palauras-chave}

desigualdade, regressão logística, Índice de Oportunidade Humana.

Classificação JEL C43, D30, D63

\section{Key words}

inequality, logistic regression, Human Opportunity Index.

JEL Classification C43, D30, D63
Helena Cristina Dill Mestre em Desenvolvimento Econômico pelo PPGDE/UFPR

Flávio de Oliveira Gonçalves Professor adjunto da Universidade Federal do Paraná

\section{Abstract}

O objetivo deste artigo é mensurar um índice de oportunidade humana para os estados brasileiros e, então, analisar as disparidades existentes no país, no que diz respeito à igualdade de oportunidade. Utiliza-se um modelo de regressão logística para estimar a probabilidade de os indivíduos terem acesso a serviços básicos, como energia elétrica, água limpa, saneamento e escola, e, a partir disso, constrói-se o Índice de Oportunidade Humana (IOH). Os resultados indicam que há grandes diferenças entre os estados brasileiros, principalmente no acesso ao saneamento, sendo esse o serviço que mais necessita de atenção das políticas sociais.
The aim of this paper is to calculate a human opportunity index for Brazilian states and then analyze the disparities in the country regarding equality of opportunity. We use a logistic regression model for the probability of individuals having access to basic services, like electricity, clean water, sanitation and schooling. Based on this, we build the Human Opportunity Index. The estimation results indicate that there are great differences between the Brazilian states, especially in the access to sanitation. Thus, this is the service that needs more attention by social policies. 


\section{1_Introdução}

A concepção de sociedade justa está estreitamente relacionada ao conceito de igualdade social. Estudos retratam a desigualdade, especialmente no que se refere aos resultados (renda), como algo indesejado e que deve ser combatido e superado por meio de políticas e incentivos públicos. Entretanto, outra linha de pesquisa, desenvolvida principalmente a partir da década de 1970 com o trabalho seminal de Ralws (1971), aponta um tipo particular de desigualdade como o socialmente injusto: a desigualdade de oportunidade.

De acordo com tal conceito, as vantagens pessoais de um indivíduo, tais como os rendimentos, são pautadas por dois componentes distintos, porém relacionados: as circunstâncias, características inatas dos indivíduos, como gênero, raça e local de nascimento, e o esforço ${ }^{1}$ exercido por cada um. O primeiro fator está além do controle do agente apesar de influenciar seus resultados. Já o segundo está diretamente subordinado às escolhas individuais. Isso posto, numa sociedade onde a igualdade de oportunidade prevalece, a desigualdade de resultados observada, como a desigualdade de renda, deve ser oriunda apenas dos diferentes esforços empregados pelos indivíduos. Ou seja, as circunstâncias pessoais não podem exercer nenhum papel para a obtenção de resultados, uma vez que, de acordo com Peragine (2004), desigualdades econômicas advindas de fatores sobre os quais os indivíduos não têm controle são injustas e devem ser compensadas pela sociedade.

Igualdade nesse sentido significa que dois indivíduos situados em uma mesma posição relativa em duas distribuiçôes distintas (por exemplo, notas em exames padronizados entre alunos de escolas privadas e públicas no Brasil) devam ter acesso às mesmas oportunidades econômicas. Assim, se a desigualdade de renda, ou outro indicador de resultado, for oriunda não apenas dos esforços dos indivíduos e estiver correlacionada com as características pessoais de cada um, haverá desigualdade de oportunidade.

A discussão teórica da visão igualitária de justiça social voltada ao conceito de desigualdade de oportunidade inicia-se no campo filosófico com estudos como Arneson (1989), Cohen (1989) e Dworkin (1981). Para além da filosofia, Roemer (1996, 1998) oferece uma formalizaçáo do conceito, em que, pela primeira vez na literatura, esforço e circunstâncias são denotados como determinantes da desigualdade social, que, por sua vez, é apresentada em duas formas, a socialmente justa, resultado de diferentes esforços, e a injusta, oriunda das circunstâncias dos indivíduos. Sua pesquisa é um marco para o início de toda uma
${ }^{1}$ Tal denominaçáo foi dada inicialmente por Roemer (1996) e será a adotada neste artigo. 
literatura voltada ao estudo da desigualdade de oportunidade, principalmente no que concerne ao desenvolvimento de instrumentos para sua mensuração.

Buscando aliar o conceito teórico à prática empírica, baseando-se em Roemer (1998), muitos estudos têm levantado questóes sobre a forma de medir a desigualdade de oportunidade, dada a dificuldade encontrada em conciliar os dados necessários à pesquisa aos que estão disponíveis, entre outros fatores complicadores. Apesar disso, várias publicações têm buscado obter uma medida para a desigualdade de oportunidade, embora não se tenha chegado a um consenso sobre qual a forma mais adequada para essa mensuração.

Lefranc, Pistolesi e Trannoy (2008) utilizam o critério de dominância estocástica como instrumento para obter uma medida de desigualdade de oportunidade. O estudo de Checchi e Peragine (2009) analisa a desigualdade de oportunidade de uma forma não paramétrica entre as regióes Norte e Centro-Sul da Itália, cujas estimativas explicam um terço da desigualdade de resultado observada. Já Bourguignon, Ferreira e Menéndez (2007) utilizam microdados para decompor as oportunidades em um efeito direto sobre os salários e outro indireto, que opera através do esforço. Apesar de utilizarem métodos diferentes, tais pesquisas apre- sentam semelhanças: obtêm medidas de desigualdade de oportunidade mediante a inclusão dos componentes circunstância e esforço.

Outra linha desenvolvida por Barros et al. (2009) se concentra na igualdade de oportunidade valendo-se somente das variáveis relacionadas às circunstâncias, uma vez que apenas a desigualdade derivada desse fator é socialmente injusta, e o impacto dos esforços não é diretamente observável. Os autores constroem o Índice de Oportunidade Humana ( $\mathrm{IOH}$ ) para o acesso a bens e serviços essenciais de pessoas com idade igual ou inferior a 16 anos. Tal índice revela quáo justo está distribuído na sociedade o acesso a oportunidades básicas como saneamento, água, energia elétrica e escola. Com base em dados de 19 países da América Latina e do Caribe, durante o período de 1995 a 2005, Barros et al. (2009) mensuram IOHs nacionais por oportunidade, de forma a comparar e a classificar os países de acordo com o nível de igualdade de oportunidade revelado pelos índices.

Tendo em vista as recentes contribuiçóes no sentido de mensurar a desigualdade de oportunidade, o objetivo desta pesquisa é, utilizando a metodologia proposta por Barros et al. (2009), desenvolver o Índice de Oportunidade Humana (IOH) para os estados brasileiros. Consi- 
derando que os trabalhos empíricos sobre desigualdade de oportunidade são escassos, esta pesquisa é inovadora no que concerne a analisar tal desigualdade para o Brasil, desmembrando o índice para os estados e, com isso, possibilitando observar as disparidades regionais do país com relação ao acesso às oportunidades. Não se tem conhecimento sobre outros textos que explorem empiricamente o $\mathrm{IOH}$ além de Barros et al. (2009) e Vega et al. (2010), ambos referentes a mesma amostra, o que denota outra contribuição do artigo para a discussão.

Considerando este objetivo, o artigo está dividido em quatro seçóes. Após esta introdução, a segunda seção trata sobre as variáveis utilizadas e o procedimento de estimação do $\mathrm{IOH}$. A terceira seção apresenta a regressão estimada e os resultados do IOH para os estados brasileiros, tanto por oportunidade quanto um $\mathrm{IOH}$ denominado geral que agrega os quatro serviços básicos em um único indicador. A última seção reporta às conclusóes do trabalho.

\section{2 _Índice de Oportunidade Humana}

Os dois componentes da denominada desigualdade de resultados, esforço e circunstância, têm sido definidos de forma alternativa na literatura como variáveis de responsabilidade e de náo responsa- bilidade. ${ }^{2}$ Apesar de rótulo distinto, o conteúdo é o mesmo: fatores pelos quais o indivíduo não é responsável, como gênero ou educação dos pais, não devem exercer influência sobre a desigualdade de resultados, pois, caso contrário, há desigualdade de oportunidade. Já fatores cuja responsabilidade está a cargo do indivíduo, como o esforço exercido por ele, podem e comumente impactam na desigualdade de resultados.

Na busca da mensuração de como essas variáveis de não responsabilidade interagem e contribuem para a formação da desigualdade no acesso às oportunidades, o Índice de Oportunidade Humana (IOH) desenvolvido por Barros et al. (2009) difere das demais aplicaçóes empíricas, no sentido de que não mensura diretamente a desigualdade de oportunidade. $\mathrm{O}$ índice é uma medida de como as oportunidades disponíveis numa sociedade, definidas como o acesso a determinados bens e serviços básicos, são alocadas com base no princípio da igualdade de oportunidade (Vega et al., 2010). Ou seja, é um índice que revela quão distante está uma sociedade de um acesso amplo e igualitário a um dado bem ou serviço.

Com o intuito de focar somente nas variáveis referentes à circunstância, $\mathrm{O}$ IOH foi desenvolvido pelos autores tendo como base microdados que incluem

\footnotetext{
2 Trabalhos como Ramos e Van de Gaer (2009) e Figueiredo e Ziegelmann (2010) utilizam os termos responsibility e non responsibility como sinônimos às definiçôes de esforço e circunstância, respectivamente.
} 
${ }^{3}$ Esta seção foi baseada em Vega et al. (2010). Para um aprofundamento algébrico das deduçóes aqui apresentadas, ver Barros et al. (2008) apenas indivíduos com idade igual ou inferior a 16 anos. De acordo com Barros et al. (2009), mensurar a igualdade de oportunidade entre agentes de tal faixa etária permite isolar a variável circunstância da de esforço e analisar os impactos somente da primeira, uma vez que indivíduos com tal idade não estão aptos a escolher seu esforço, e, portanto, as diferenças observadas no acesso que eles têm a bens e serviços básicos decorrem de suas características pessoais e de seu ambiente. Outro ponto a ser ressaltado com relação à análise para crianças é que intervençóes para igualar oportunidades quando o indivíduo ainda é jovem são menos custosas e mais custo-efetivas do que intervenções na fase adulta (Vega et al., 2010).

$\mathrm{O} \mathrm{IOH}$ varia numa escala de zero a 100, e, quanto mais próximo de 100 é a desigualdade em seu acesso, há mais oportunidades disponíveis e menos correlacionadas às circunstâncias pessoais.

O índice é construído através da combinação de dois elementos. O primeiro é a taxa de cobertura de um dado bem ou serviço, que revela a proporçáo da população que tem acesso à determinada oportunidade, ou seja, a quantidade de oportunidade disponível. O segundo é a desigualdade de oportunidade, dada pelo índice de dissimilaridade, índice bastante utilizado em Sociologia, que, aplicado ao estudo em questão, fornece uma medida de como a taxa de cobertura difere entre grupos de indivíduos classificados de acordo com suas circunstâncias.

\section{1_Método de cálculo ${ }^{3}$}

A probabilidade de um indivíduo ter acesso a um dado bem ou serviço pode ser estimada a partir de uma relação empírica entre o conjunto de circunstâncias pessoais e o acesso efetivo que ele tem a essa oportunidade. A forma com que essa relação empírica se dá pode ser explicitada mediante um modelo de regressão logística, no qual é regredido o acesso contra o grupo de circunstâncias, o que possibilita estimar a probabilidade de o indivíduo ter acesso à oportunidade dado que possui determinadas características pessoais.

De forma mais específica, a probabilidade $(\mathrm{P})$ de um agente i ter acesso $(A=1)$ a oportunidade em questão está condicionada ao vetor $\mathrm{k}$-dimensional das circunstâncias (x). Essa relação pode ser expressa como:

$P\left(A=1 \mid x_{1 i} \cdots x_{m i}\right)$

onde $\mathrm{i}=1, \ldots, \mathrm{n}$ e $\left\{\mathrm{x}_{\mathrm{k}} \mid \mathrm{k}=1, \ldots, \mathrm{m}\right\}$ e onde $A=1$ simboliza se a pessoa tem acesso e $\mathrm{A}=0$ se não tem. 
Dado que o acesso à oportunidadeé uma função do conjunto de circunstâncias do indivíduo, o procedimento da regressão logística permite obter os parâmetros de tal função. Formalmente, são estimados os $\beta$ s da seguinte regressão:

$$
\begin{aligned}
\frac{P\left(A=1 \mid x_{1 i} \cdots x_{m i}\right)}{1-P\left(A=1 \mid x_{1 i} \cdots x_{m i}\right)} & = \\
& =e^{\beta_{o}+\sum_{k=1}^{m} x_{k i} \beta_{k}}
\end{aligned}
$$

Entretanto, o resultado estimado dessa regressão ainda não reporta a probabilidade em si, uma vez que o procedimento logístico é feito por meio da utilização de uma função de máxima verossimilhança. Assim, procede-se à seguinte operação para que a probabilidade individual possa ser obtida:

$$
p_{i}=\left\{\frac{e^{\beta_{o}+\sum_{k=1}^{m} x_{k i} \beta_{k}}}{1+e^{\beta_{o}+\sum_{k=1}^{m} x_{k i} \beta_{k}}}\right\}
$$

Tendo estimado as probabilidades para todos os indivíduos da amostra de tamanho N, a taxa de cobertura de dado bem ou serviço é mensurada como a média simples dessas probabilidades de acesso condicionais dos indivíduos à oportunidade em questão. Tem-se, assim, uma taxa de acesso média da população como um todo para uma oportunidade específica:

$$
C=\frac{1}{N} \sum_{i=1}^{n} p_{i}
$$

onde $\mathrm{C}$ representa a taxa de cobertura, indicador que tem seu valor situado no intervalo entre zero e um, sendo maior a cobertura existente de determinado serviço quanto mais próximo de um for seu valor.

A taxa de cobertura é um importante indicador, e várias metas de universalização de acesso a bens e serviços básicos têm sido baseadas na conquista de um aumento desse indicador. Entretanto, a alocação desses bens e serviços não é aleatória, nem igualitária (Vega et al., 2010). Por mais que se aumente a taxa de cobertura, ela não será alocada de forma igualitária entre os diversos indivíduos, uma vez que as características pessoais são fatores relevantes para essa alocação. Assim, torna-se necessário uma medida que revele o grau com que as oportunidades são distribuídas entre os diferentes grupos de circunstâncias. É com esse intuito que se inclui na análise o índice de dissimilaridade. 


\footnotetext{
${ }^{4}$ Uma interessante extensão da aplicaçáo do índice de dissimilaridade ao tema da desigualdade de oportunidade pode ser consultada em Yalonetzky (2010).

${ }^{5}$ Calculado dessa maneira, o valor do $\mathrm{IOH}$ estará entre zero e um. Entretanto, ele será apresentado neste trabalho num intervalo entre zero e 100 para facilitar a visualização dos resultados.
}

O índice de dissimilaridade ${ }^{4}$ mede as diferenças das taxas de acesso à certa oportunidade para grupos de indivíduos definidos por circunstâncias comparadas com o acesso médio apresentado pela taxa de cobertura. Pode ser interpretado como a fração de todas as oportunidades que precisam ser realocadas para restaurar a igualdade de oportunidade (Barros et al., 2009). Fornece, portanto, uma medida da desigualdade de oportunidade.

Esse índice de desigualdade de oportunidade pode ser obtido a partir da equação:

$$
D=\frac{1}{2 C} \sum_{i=1}^{n} \frac{1}{N}\left|p_{i}-C\right|
$$

Seu valor varia entre zero e um, e, quanto mais próximo de zero, mais igualitária e justa na distribuição do acesso à oportunidade sob análise é a sociedade em questão. Assim, quando maior o valor de $\mathrm{D}$, maior a diferença no acesso grupo-específico para um bem relativamente à taxa de cobertura desse bem.

$\mathrm{O}$ Índice de Oportunidade $\mathrm{Hu}$ mana é obtido combinando-se a taxa de cobertura e o índice D. Como o índice de dissimilaridade representa a desigualdade de oportunidade existente, tem-se que (1-D) pode ser interpretado como a porcentagem de oportunidades que são alocadas de forma igualitária (Vega et al., 2010). Partindo-se dessa visão, o $\mathrm{IOH}$ pode ser expresso como5:

$$
I O H=C *(1-D)
$$

De forma intuitiva, o $\mathrm{IOH}$ assim calculado pondera a taxa de cobertura da oportunidade pela proporção dessa que é distribuída de maneira socialmente justa. Isto é, capta somente a cobertura do bem ou serviço que é adequadamente alocada entre os diversos grupos de circunstâncias. Se não houver diferenças de acesso aos diversos grupos de circunstâncias, ou seja, se o acesso dos indivíduos a determinada oportunidade é independente de suas características pessoais, $\mathrm{D}$ assume o valor zero e o IOH será igual à taxa de cobertura.

Outra forma de expressar o IOH é:

$$
\begin{aligned}
& I O H=C *(1-D) \Rightarrow I O H= \\
& =C-C * D \Rightarrow I O H=C-P
\end{aligned}
$$

onde $\mathrm{P}=\mathrm{C}^{*} \mathrm{D}$ representa a penalidade imposta sobre taxa de cobertura pela desigualdade de oportunidade.

O estudo de Barros et al. (2009) abrange 19 países da América Latina e do Caribe, durante o período de 1995 a 2005. Os autores constroem um IOH para ca- 
da oportunidade relacionada à habitação, como saneamento e acesso à eletricidade e água limpa, e também para cada oportunidade relacionada à educaçáo, como frequência escolar e conclusão da sexta série na idade adequada. Os resultados denotam que muitos países estão próximos do acesso universal à eletricidade, apesar de existirem alguns com baixo $\mathrm{IOH}$ para esse item. A América Latina como um todo apresenta $\mathrm{IOH}$ muito pior para o saneamento do que para o acesso à água, com média regional do $\mathrm{IOH}$ de 67 para a água e de apenas 43 para o saneamento. $\mathrm{O} \mathrm{IOH}$ para frequência escolar é alto em todos os países, com média regional de 90 , e, para a conclusão da sexta série, a média é 62 , valor considerado baixo e que apresenta elevada dispersão entre os países.

\section{2_Especificação das variáveis e da base de dados}

A base de dados utilizada neste estudo foram os microdados da Pesquisa Nacional por Amostras de Domicílios (PNAD) do ano de 2009. Tendo em vista o objetivo de isolar e analisar somente a influência das circunstâncias ao acesso às oportunidades, foram considerados somente indivíduos com idade igual ou inferior a 16 anos.

As variáveis selecionadas para compor as oportunidades, isto é, bens e serviços básicos cujo acesso pode desempenhar pa- pel essencial na vida adulta, foram o acesso à eletricidade, acesso ao saneamento adequado, acesso à água canalizada, que são dimensões relativas à habitação e são relacionadas à qualidade de vida, e a probabilidade de se completar a sexta série na idade correta, dimensáo que tenta captar a oportunidade de educação básica (Barros et al., 2009). As oportunidades foram especificadas de forma binária, assumindo o valor um se o indivíduo tem acesso ao bem ou serviço e zero se náo tem.

O acesso à eletricidade é uma porta de entrada para o acesso a outras oportunidades, tais como o acesso à informação via internet e televisão, e permite também estudos no período noturno. Para efeitos da análise, foram considerados como de efetivo acesso apenas os domicílios que possuem energia elétrica como forma de iluminação.

Os acessos à água limpa e ao saneamento estão relacionados principalmente à saúde. Segundo World Bank (2006), água limpa, sistemas de saúde e saneamento básico são os determinantes mais importantes da esperança de vida ao nascer. Para o acesso à água limpa, foram considerados os domicílios que possuem água canalizada em pelo menos um cômodo, independentemente se a proveniência é a rede geral de distribuição, poço ou nascente. Com relação ao saneamento, foi utiliza- 
da a forma de escoadouro do domicílio, sendo considerados os que apresentam rede de esgoto ou fossa ligada à rede de esgoto como os que têm acesso a essa oportunidade.

Para avaliar como as circunstâncias impactam na probabilidade de os indivíduos terem acesso às oportunidades descritas, foram consideradas as seguintes características: gênero e raça do indivíduo, que capturam efeitos de discriminação direta; área de residência, para confrontar as disparidades entre as áreas urbana $\mathrm{e}$ rural; gênero da pessoa de referência, isto é, da pessoa declarada como responsável pelo domicílio quando da realização da pesquisa, para discriminação indireta; presença da mãe, para indícios de estrutura familiar, ${ }^{6}$ educação da pessoa de referência do domicílio, como uma proxy para origem familiar, ${ }^{7}$ renda mensal do-

\footnotetext{
${ }^{6}$ A variável mais adequada seria a presença de ambos os pais, como utilizam Barros et al. (2008), mas os dados disponíveis não permitem tal compilação.

${ }^{7}$ Educação de ambos os pais teve importante impacto na análise feita por Bourguignon, Ferreira e Menéndez (2007), mas a insuficiência de dados novamente impede uma análise mais completa. A
}

\author{
única fonte desse dado para \\ o Brasil é o suplemento da \\ PNAD de 1996, base de dados \\ utilizada pelos autores. \\ ${ }^{8} \mathrm{~A}$ indicação sobre quais \\ variáveis foram incluídas, sua \\ nomenclatura na equação (8) \\ adiante, suas especificaçóes, \\ bem como as classes que \\ foram consideradas como \\ referência para as binárias, \\ estão denotadas no Anexo.
}

miciliar per capita, para captar o efeito dos recursos disponíveis a que se tem acesso; e número de pessoas que vivem no domicílio.

A especificação das variáveis seguiu a abordagem de Barros et al. (2008), de acordo com a necessidade do modelo: ${ }^{8}$ binária para gênero, raça, área de residência, presença da mãe e gênero da pessoa de referência, logarítmica para renda mensal domiciliar per capita, linear para o número de pessoas na família e quadrática para educação da pessoa de referência. Apesar das especificações, as funções continuam lineares nos parâmetros. Para construir uma dummy para raça, as cinco categorias apresentadas na PNAD foram englobadas em apenas duas, seguindo o mesmo critério de Bourguignon, Ferreira e Menéndez (2007), entre as quais a primeira inclui indivíduos brancos e amarelos e a segunda inclui pretos, pardos e indígenas, caracterizando as categorias brancos e não brancos.

Após a seleção da faixa etária requerida pelo estudo e a exclusão de variáveis que não continham valor em seus respectivos campos, a amostra resultou num total de 108.357 indivíduos para os 26 estados brasileiros mais o Distrito Federal. Foram também desconsiderados indivíduos sem informaçóes sobre a série que frequentavam. Cabe mencionar que, para a obtenção das estimativas da regressão 
logística, foram utilizados os pesos estatísticos da pessoa indicados na PNAD, possibilitando, desta maneira, expandir os resultados obtidos na amostra para a população brasileira como um todo.

\section{3_Estimação e resultados para o IOH}

Valendo-se das oito circunstâncias como variáveis dependentes para explicar a probabilidade de acesso a quatro bens e serviços básicos para indivíduos de até 16 anos de idade, foi utilizado um modelo de regressão logística para estimar a seguinte regressão:

$$
\begin{aligned}
& O p_{i j}=\exp \left(\beta_{o}+\beta_{1} \text { sexo }_{i}+\beta_{2} \text { raça }_{i}+\right. \\
& +\beta_{3} \text { urbano }_{i}+\beta_{4} \text { gen }_{-} \text {pesref }_{i}+ \\
& +\beta_{5} \text { presenca }_{-} \text {mae }_{i}+\beta_{6} \text { educ }_{-} \text {pesref }_{i}+ \\
& \left.+\beta_{7} \text { lnrenda }_{i}+\beta_{8} \text { num }_{-} \text {pes }_{i}\right)
\end{aligned}
$$

em que:

$$
O p_{i j}=\frac{P\left(A_{j}=1 \mid x_{k i}\right)}{1-P\left(A_{j}=1 \mid x_{k i}\right)}
$$

onde Op representa o acesso pelo indivíduo i à oportunidade $\mathrm{j}$, sendo que $\mathrm{j}$ $=1, \ldots, 4$, simbolizando as quatro oportunidades objeto deste estudo (água, energia, saneamento e escola), e $i=1, \ldots, n$.
A regressão foi realizada para cada oportunidade, seguindo a especificação das variáveis apresentada na seção 2.2 , resultando nos seguintes coeficientes e razóes de chances (odds ratios) veja Tabela 1.

Apesar de os coeficientes da variável sexo nas regressões de acesso à água, à energia elétrica e ao saneamento não serem estatisticamente significativas, optou-se por mantê-la no modelo para a padronização da análise, uma vez que todas as variáveis são significativas a $1 \%$ na explicaçáo do acesso à escola. Além disso, o resultado do teste $\chi 2$ de Wald sobre a significância global do modelo é estatisticamente significativo a $1 \%$ para as quatro regressóes realizadas.

Analisando de maneira geral a relaçấo entre o acesso às oportunidades $\mathrm{e}$ as circunstâncias incluídas no modelo, as razóes de chances (odds ratios) mostram que a variável sexo quase não afeta o acesso às oportunidades água, energia e saneamento, tendo impacto maior apenas no acesso à escola, em que o fato de o indivíduo ser do sexo masculino reduz em $14,6 \%$ sua probabilidade de acesso ${ }^{9}$. Esse resultado pode indicar maior custo de oportunidade para os meninos em frequentar a escola. Já com relação à raça, ser classificado como branco aumenta a probabilidade de acesso a todas as oportunidades, chegando a elevar em cerca de $91 \%$ o acesso no caso da água. O fato

\footnotetext{
${ }^{9}$ As interpretaçóes aqui apresentadas referem-se aos coeficientes e razóes de chances estimados com base nos impactos das variáveis explicativas sobre as probabilidades de acesso aos serviços analisados, não implicando causalidade dos regressores sobre os índices posteriormente apresentados.
} 
Tabela 1 - Coeficientes estimados e odds ratios obtidos pela regressão logística, para cada oportunidade

\begin{tabular}{|c|c|c|c|c|c|c|c|c|}
\hline & $\begin{array}{l}\text { Água } \\
\text { Coef. }\end{array}$ & $\begin{array}{l}\text { Odds } \\
\text { Ratio }\end{array}$ & $\begin{array}{l}\text { Energia } \\
\text { Coef. }\end{array}$ & $\mid \begin{array}{l}\text { Odds } \\
\text { Ratio }\end{array}$ & $\begin{array}{l}\text { Saneamentc } \\
\text { Coef. }\end{array}$ & Odds & $\begin{array}{l}\text { Escola } \\
\text { Coef. }\end{array}$ & $\begin{array}{l}\text { Odds } \\
\text { Ratio }\end{array}$ \\
\hline constante & $\begin{array}{l}-3,895^{*} \\
(0,103)\end{array}$ & - & $\begin{array}{l}-0,078 \\
(0,195)\end{array}$ & - & $\begin{array}{l}-5,983^{*} \\
(0,077)\end{array}$ & - & $\begin{array}{c}0,864^{*} \\
(0,065)\end{array}$ & - \\
\hline sexo & $\begin{array}{l}-0,009 \\
(0,025)\end{array}$ & 0,991 & $\begin{array}{l}-0,020 \\
(0,053)\end{array}$ & 0,980 & $\begin{array}{l}-0,008 \\
(0,015)\end{array}$ & 0,992 & $\begin{array}{l}-0,158^{*} \\
(0,015)\end{array}$ & 0,854 \\
\hline raça & $\begin{array}{c}0,650^{*} \\
(0,029)\end{array}$ & 1,916 & $\begin{array}{r}0,552^{*} \\
(0,065)\end{array}$ & 1,736 & $\begin{array}{c}0,459^{*} \\
(0,016)\end{array}$ & 1,583 & $\begin{array}{c}0,213^{*} \\
(0,016)\end{array}$ & 1,237 \\
\hline urbano & $\begin{array}{c}2,062^{*} \\
(0,026)\end{array}$ & 7,860 & $\begin{array}{c}2,641^{*} \\
(0,073)\end{array}$ & 14,025 & $\begin{array}{c}2,720^{*} \\
(0,036)\end{array}$ & 15,180 & $\begin{array}{c}0,204^{*} \\
(0,020)\end{array}$ & 1,226 \\
\hline gen_pesref & $\begin{array}{l}-0,217^{*} \\
(0,031)\end{array}$ & 0,805 & $\begin{array}{c}-0,147^{* *} \\
(0,071)\end{array}$ & 0,863 & $\begin{array}{l}-0,234^{*} \\
(0,017)\end{array}$ & 0,791 & $\begin{array}{c}0,232^{*} \\
(0,017)\end{array}$ & 1,262 \\
\hline presenca_mae & $\begin{array}{r}0,311^{*} \\
(0,041)\end{array}$ & 1,364 & $\begin{array}{r}0,443^{*} \\
(0,086)\end{array}$ & 1,558 & $\begin{array}{c}0,249^{*} \\
(0,026)\end{array}$ & 1,283 & $\begin{array}{c}0,436^{*} \\
(0,025)\end{array}$ & 1,547 \\
\hline educ_pesref & $\begin{array}{c}0,007^{*} \\
(0,0003)\end{array}$ & 1,007 & $\begin{array}{r}0,003^{*} \\
(0,0007)\end{array}$ & 1,003 & $\begin{array}{r}0,0023^{*} \\
(0,0001)\end{array}$ & 1,002 & $\begin{array}{r}0,0034^{*} \\
(0,0001)\end{array}$ & 1,003 \\
\hline lnrenda & $\begin{array}{c}0,822^{*} \\
(0,016)\end{array}$ & 2,275 & $\begin{array}{r}0,554^{*} \\
(0,030)\end{array}$ & 1,741 & $\begin{array}{c}0,579^{*} \\
(0,011)\end{array}$ & 1,785 & $\begin{array}{r}-0,026^{*} \\
(0,0097)\end{array}$ & 0,974 \\
\hline num_pes & $\begin{array}{l}-0,052^{*} \\
(0,008)\end{array}$ & 0,950 & $\begin{array}{l}-0,127^{*} \\
(0,014)\end{array}$ & 0,881 & $\begin{array}{c}-0,013^{* *} \\
(0,005)\end{array}$ & 0,987 & $\begin{array}{l}-0,155^{*} \\
(0,005)\end{array}$ & 0,856 \\
\hline Log pseudolikelihood & \multicolumn{2}{|c|}{$-25.853,244$} & \multicolumn{2}{|r|}{$-7.206,003$} & \multicolumn{2}{|c|}{$-59.242,383$} & \multicolumn{2}{|c|}{$-60.829,389$} \\
\hline Wald $\chi^{2}$ & \multicolumn{2}{|c|}{$13.073,800$} & \multicolumn{2}{|r|}{$2.845,020$} & \multicolumn{2}{|c|}{$14.347,740$} & \multicolumn{2}{|r|}{$3.028,940$} \\
\hline Pseudo R2 & & 0,303 & & 0,259 & & 0,211 & & 0,031 \\
\hline
\end{tabular}

$\left({ }^{*}\right)$ Valores significativos a $\left.1 \% .{ }^{* *}\right)$ Valores significativos a $5 \%$. Desvio-padráo entre parênteses.

Fonte: elaboração própria. 
de residir em localidade urbana também apresenta impacto positivo no acesso, sendo esta a circunstância com a maior razão de chances em todas as oportunidades com exceção da escola.

A razão de chances para o gênero da pessoa de referência traz certa curiosidade sobre seu efeito, uma vez que pessoas de referência do sexo masculino têm influência negativa no acesso à água, à energia e ao saneamento, reduzindo de $13 \%$ a $20 \%$ aproximadamente a probabilidade de acesso. Entretanto, para a oportunidade escola, o fato de a pessoa de referência ser do sexo masculino aumenta em cerca de $26 \%$ a probabilidade de acesso do indivíduo sob análise. A presença da mãe no domicílio apresenta impacto positivo, elevando de $28 \%$ a $55 \%$ a probabilidade de os indivíduos terem acesso às oportunidades. $\mathrm{O}$ nível de educação da pessoa de referência também possui influência positiva, mas bem menor do que o efeito da presença da mãe, sendo essa influência de menos de $1 \%$ sobre o acesso às oportunidades.

A variável renda apresentou impacto negativo apenas sobre $\mathrm{o}$ acesso à escola, porém de magnitude mínima; para as demais oportunidades, seu impacto é positivo, variando de $74 \%$ a $127 \%$ sobre a probabilidade de acesso. Tais resultados não esperados para as variáveis renda e gê- nero da pessoa de referência na regressão do acesso à escola podem indicar que a dimensão do fator educação é bem maior do que se tenta capturar neste estudo. Isso no sentido de que há uma literatura ampla e especializada no tema educação, que apresenta especificidades maiores do que as incluídas na análise, sendo esse processo mais complexo do que o aqui considerado. Com relação ao número de pessoas no domicílio, o efeito sobre as probabilidades de acesso de todas as oportunidades foi negativo, afetando desfavoravelmente de $1 \%$ a $14 \%$ a probabilidade de acesso.

Dados os critérios de seleçáo da amostra utilizada no presente estudo, em que são considerados apenas indivíduos até 16 anos, e as variáveis explicativas referentes a circunstâncias, que são fatores fora do controle dos indivíduos, espera-se que a correlação entre as variáveis independentes não seja elevada a ponto de ocasionar problemas na elaboração dos índices propostos. No Anexo é apresentada tabela que contém os coeficientes de correlação entre as variáveis, onde se nota associação mínima entre a maioria delas, inevitável, uma vez que a multicolinearidade é característica inerente à maioria dos problemas abordados em regressóes.

Quanto à possibilidade de causalidade reversa entre o acesso às oportunidades e as variáveis independentes, vale ressaltar 
que, como para o caso de multicolinearidade, a amostra selecionou indivíduos de tal faixa etária que as explicativas são todas exógenas a ele. Ou seja, o fato de o indivíduo ter acesso aos serviços aqui considerados não deve exercer influência direta sobre o conjunto de suas circunstâncias pessoais. O efeito reverso do acesso a essas oportunidades sobre as variáveis renda, número de pessoas no domicílio e educação da pessoa de referência, pode ocorrer, mas se daria para os indivíduos em sua fase adulta. Ou seja, para pais, familiares ou responsáveis pela criação dos indivíduos aqui analisados, o acesso à água, à energia elétrica, ao saneamento e à escola podem influenciar sua renda, educação ou o número de pessoas que vivem em seu domicilio. Considera-se, porém, que, para os indivíduos que compóem a amostra desta pesquisa, tais variáveis são exógenas, e sua probabilidade de acesso não terá impactos na sua renda ou nos demais regressores, uma vez que essas características são impostas a eles.

A presença de multicolinearidade ou a ocorrência de causalidade reversa impactariam na estimação das probabilidades de acesso pela regressão logística. $\mathrm{Na}$ medida em que a base primordial para o cálculo dos índices de desigualdade de oportunidade, de oportunidade humana e taxa de cobertura são essas probabilidades previstas pelo modelo, a presença de algum desses problemas econométricos afetaria os índices através desse canal. Todavia, como destacado anteriormente, esses não constituem entraves à análise aqui proposta. A causalidade ocorre, portanto, com base nas variáveis independentes para as probabilidades de acesso estimadas, sendo que as circunstâncias influenciam indiretamente os índices apresentados através de seu impacto direto sobre as probabilidades de acesso às oportunidades.

Valendo-se das regressóes estimadas, foram obtidas as probabilidades de acesso de cada indivíduo aos diferentes bens e serviços sob análise. Utilizando-se essas probabilidades, foi procedido o cálculo conforme descrito na seção 2.1 para a obtenção do $\mathrm{IOH}$ de cada oportunidade para os estados brasileiros.

\section{1_IOH geral para os estados brasileiros}

Com vistas a uma análise mais abrangente da igualdade de oportunidade entre os estados brasileiros, calculou-se inicialmente um Índice de Oportunidade $\mathrm{Hu}$ mana geral, indicador que agrega as quatro oportunidades em uma única medida. Para tanto, foi utilizada a média simples das probabilidades individuais de acesso estimadas por meio da regressão logística, procedendo-se, então, ao cálculo do IOH como descrito na sessão anterior. A 
intenção é verificar como está a distribuição conjunta das oportunidades alocadas como base na igualdade de oportunidade em todas as regióes brasileiras. Os resultados são reportados na Tabela 2.

Os valores do $\mathrm{IOH}$ geral mostram que há grandes disparidades na distribuição equitativa do acesso às oportunidades em todo o território, considerando que varia no intervalo entre zero e cem, bem como a taxa de cobertura e o índice de dissimilaridade, tal como ressaltado na sessão dois. Os estados onde há mais oportunidades e onde essas são distribuídas de forma igualitária são Rio de Janeiro, São Paulo e o Distrito Federal. Também são esses os que possuem os menores índices de desigualdade de oportunidade. A regiáo Sul e parte da região Sudeste concentram os estados que apresentam tanto os menores índices de desigualdade de oportunidade quanto os maiores IOHs. Em contraposição, é nas regiôes Norte e Nordeste que se observam os menores Índices de Oportunidade Humana e da taxa de cobertura e os maiores índices de dissimilaridade, sendo Acre, Piauí e Maranhão os estados que despontam com os piores indicadores.

\section{2_IOH estadual por oportunidade}

Objetivando analisar de modo minucioso a igualdade de oportunidade entre os esta- dos, a Tabela 3, a seguir, apresenta os resultados obtidos para o Índice de Oportunidade Humana, o índice de dissimilaridade e a taxa de cobertura por oportunidade e por estado. Ressalte-se que os índices variam entre zero e cem.

Analisando os resultados para o acesso à água encanada, sua taxa de cobertura é mais elevada se comparada com o mesmo indicador para o acesso ao saneamento e à escola. Apesar dessa constatação, a diferença observada na quantidade disponível da oportunidade entre os estados brasileiros é palpável: enquanto alguns contam com uma universalização próxima no acesso ao serviço, com cobertura de 95\%, outros possuem cobertura em torno de $76 \%$. O IOH dos estados apresenta-se num intervalo de 65 a 93, revelando grande disparidade entre as regióes do país. A desigualdade de oportunidade, tal como dada pelo índice de dissimilaridade, revela que, enquanto em alguns estados o valor do indicador é de 2 , em outros a influência das características pessoais no acesso ao serviço é maior, como demonstrado pelo valor de 14 para o índice. Acre e Piauí possuem os menores valores para a igualdade de oportunidade, e São Paulo, Rio de Janeiro e Distrito Federal, os maiores. Na comparação entre regióes, Sul, Sudeste e Centro-Oeste se classificam com os melhores indicadores. 
Tabela 2 - Índice de Oportunidade Humana e índice de dissimilaridade por estado brasileiro

\begin{tabular}{|c|c|c|c|c|}
\hline Região & Estado & IOH Geral & D Geral & C Geral \\
\hline \multirow{7}{*}{ Norte } & Rondônia & 68,63 & 7,08 & 73,86 \\
\hline & Acre & 60,21 & 10,55 & 67,32 \\
\hline & Amazonas & 70,64 & 5,87 & 75,05 \\
\hline & Roraima & 73,75 & 4,81 & 77,48 \\
\hline & Pará & 68,38 & 6,8 & 73,37 \\
\hline & Amapá & 66,79 & 7,41 & 72,14 \\
\hline & Tocantins & 68,54 & 7,37 & 73,99 \\
\hline \multirow{9}{*}{ Nordeste } & Maranhão & 62,65 & 8,85 & 68,73 \\
\hline & Piauí & 61,33 & 9,46 & 67,74 \\
\hline & Ceará & 70,18 & 6,27 & 74,89 \\
\hline & Rio Grande do Norte & 67,4 & 7,43 & 72,81 \\
\hline & Paraíba & 68,15 & 6,92 & 73,23 \\
\hline & Pernambuco & 69,58 & 6,56 & 74,47 \\
\hline & Alagoas & 61,54 & 9,69 & 68,15 \\
\hline & Sergipe & 69,59 & 6,49 & 74,42 \\
\hline & Bahia & 66,72 & 7,63 & 72,24 \\
\hline \multirow{4}{*}{ Sudeste } & Minas Gerais & 74,72 & 4,97 & 78,63 \\
\hline & Espírito Santo & 73,24 & 5,4 & 77,42 \\
\hline & Rio de Janeiro & 79,29 & 3,49 & 82,16 \\
\hline & São Paulo & 79,93 & 3,48 & 82,82 \\
\hline \multirow{3}{*}{ Sul } & Paraná & 77,44 & 4,63 & 81,21 \\
\hline & Santa Catarina & 78,11 & 4,79 & 82,05 \\
\hline & Rio Grande do Sul & 78,21 & 4,22 & 81,66 \\
\hline \multirow{4}{*}{ Centro-Oeste } & Mato Grosso do Sul & 75,46 & 4,86 & 79,32 \\
\hline & Mato Grosso & 73,93 & 5,32 & 78,09 \\
\hline & Goiás & 76,33 & 4,22 & 79,69 \\
\hline & Distrito Federal & 79,18 & 3,92 & 82,42 \\
\hline \multirow{2}{*}{ Variação Brasil } & Máximo & 79,93 & 10,55 & 82,82 \\
\hline & Mínimo & 60,21 & 3,48 & 67,32 \\
\hline
\end{tabular}


Com relação ao acesso à energia elétrica, todos os estados apresentam elevado índice de acesso à oportunidade, com o valor do $\mathrm{IOH}$ variando de 92 a 99 . O baixo valor do índice de desigualdade de oportunidade para a energia revela a quase universalização do acesso a esse serviço, ou seja, alta taxa de cobertura, o que é devido, em grande parte, às metas estabelecidas por agências governamentais de ampliar o acesso a toda a população. Acre e Alagoas são os estados que apresentam os menores IOHs para o acesso à energia, possuindo também os maiores índices de desigualdade de oportunidade para esse serviço básico. Novamente Rio de Janeiro, São Paulo e Brasília apresentam tanto os menores índices de desigualdade de oportunidade quanto as maiores coberturas do serviço corrigidas para a equidade.

A precariedade da oferta de sistemas de saneamento se reflete em grandes diferenças entre os estados quando se observam os indicadores para tal serviço. $\mathrm{O}$ valor do $\mathrm{IOH}$ de alguns estados para esse item chega próximo de 20 , indicando que aproximadamente $20 \%$ das oportunidades necessárias para assegurar o acesso universal ao saneamento estão disponíveis e são distribuídas de forma justa. Pela análise dos dados, o saneamento é um serviço que está muito longe de estar ao alcance de todos e é o serviço que precisa da maior aten- ção dos formuladores de políticas. Além de lidar com a insuficiente oferta dessa oportunidade, deve-se atentar para sua alocação, altamente desigual e fortemente correlacionada com as circunstâncias pessoais dos indivíduos.

Os estados de São Paulo e Rio de Janeiro mais o Distrito Federal, que se classificam como os melhores no acesso a tal oportunidade, apresentam $\mathrm{IOH}$ em torno de apenas 53. Piauí e Acre se classificam como os estados que apresentam menos oportunidades de acesso ao saneamento, com valores de 19 e 20, respectivamente, para o Índice de Oportunidade Humana. Apesar de estarem situados na região Norte, que conjuntamente com o Nordeste apresentam os piores indicadores, os estados do Amazonas e de Roraima possuem índices de desigualdade de oportunidade próximos ou mesmo menores do que estados como Santa Catarina, Rio Grande do Sul e Mato Grosso, que se localizam nas regióes com melhores indicadores.

$\mathrm{O}$ acesso ao saneamento, analisado tanto pela sua taxa de cobertura quanto pelo seu índice de desigualdade de oportunidade, revela-se alarmante em todo o país. Além de terem pouca disponibilidade do serviço, os estados apresentam grande índice de desigualdade de oportunidade, o que mostra que as circuns- 
Tabela 3 - Índice de Oportunidade Humana e índice de desigualdade de oportunidade para o acesso à energia, água, saneamento e escola

\begin{tabular}{|c|c|c|c|c|c|c|c|c|c|c|c|c|c|}
\hline Região & Estado & $\begin{array}{l}\text { IOH } \\
\text { água }\end{array}$ & D água & $\begin{array}{l}\text { Tx cob } \\
\text { água }\end{array}$ & $\begin{array}{l}\text { IOH } \\
\text { energia }\end{array}$ & $\begin{array}{l}\text { D } \\
\text { energia }\end{array}$ & $\begin{array}{l}\text { Tx cob. } \\
\text { energia }\end{array}$ & $\begin{array}{l}\text { IOH } \\
\text { saneam. }\end{array}$ & $\begin{array}{l}\text { D } \\
\text { saneam. }\end{array}$ & $\begin{array}{l}\text { Tx cob. } \\
\text { saneam. }\end{array}$ & $\begin{array}{l}\text { IOH } \\
\text { escola }\end{array}$ & D & $\begin{array}{l}\text { Tx cob. } \\
\text { escola }\end{array}$ \\
\hline \multirow{7}{*}{ Norte } & Rondônia & 79,89 & 7,61 & 86,47 & 96,38 & 1,30 & 97,72 & 30,56 & 27,74 & 42,29 & 65,37 & 5,23 & 68,98 \\
\hline & Acre & 65,53 & 14,11 & 76,30 & 92,21 & 2,87 & 94,94 & 20,66 & 38,16 & 33,42 & 60,01 & 7,13 & 64,63 \\
\hline & Amazonas & 82,85 & 6,23 & 88,36 & 96,86 & 1,25 & 98,08 & 37,56 & 18,63 & 46,17 & 63,41 & 6,15 & 67,57 \\
\hline & Roraima & 87,38 & 4,34 & 91,35 & 98,05 & 0,74 & 98,78 & 43,15 & 15,74 & 51,21 & 64,70 & 5,65 & 68,58 \\
\hline & Pará & 79,17 & 7,81 & 85,88 & 96,04 & 1,56 & 97,57 & 32,17 & 23,25 & 41,91 & 64,21 & 5,75 & 68,13 \\
\hline & Amapá & 77,92 & 8,21 & 84,90 & 95,65 & 1,65 & 97,26 & 29,06 & 27,09 & 39,86 & 62,46 & 6,11 & 66,53 \\
\hline & Tocantins & 78,60 & 8,50 & 85,91 & 95,63 & 1,75 & 97,34 & 33,41 & 24,43 & 44,22 & 64,41 & 5,99 & 68,51 \\
\hline \multirow{9}{*}{ Nordeste } & Maranhão & 69,35 & 12,06 & 78,87 & 93,71 & 2,40 & 96,01 & 23,67 & 31,03 & 34,32 & 61,47 & 6,47 & 65,73 \\
\hline & Piauí & 66,43 & 13,12 & 76,47 & 93,58 & 2,27 & 95,77 & 19,63 & 37,59 & 31,46 & 63,57 & 5,50 & 67,27 \\
\hline & Ceará & 80,47 & 7,35 & 86,86 & 96,59 & 1,36 & 97,93 & 35,56 & 20,59 & 44,78 & 66,25 & 5,31 & 69,97 \\
\hline & $\begin{array}{r}\text { Rio Grande } \\
\text { do Norte }\end{array}$ & 77,18 & 8,58 & 84,43 & 95,86 & 1,58 & 97,40 & 28,76 & 27,65 & 39,76 & 65,91 & 5,40 & 69,67 \\
\hline & Paraíba & 77,89 & 8,23 & 84,88 & 96,09 & 1,53 & 97,58 & 31,40 & 23,68 & 41,15 & 65,48 & 5,49 & 69,28 \\
\hline & Pernambuco & 79,77 & 7,59 & 86,33 & 96,44 & 1,41 & 97,83 & 34,27 & 21,88 & 43,87 & 66,02 & 5,51 & 69,87 \\
\hline & Alagoas & 66,32 & 13,64 & 76,80 & 92,49 & 2,90 & 95,26 & 22,54 & 32,92 & 33,60 & 62,78 & 6,18 & 66,93 \\
\hline & Sergipe & 79,25 & 7,95 & 86,10 & 96,20 & 1,54 & 97,71 & 35,13 & 20,74 & 44,32 & 65,95 & 5,21 & 69,57 \\
\hline & Bahia & 75,53 & 9,53 & 83,49 & 95,42 & 1,79 & 97,17 & 29,78 & 25,98 & 40,23 & 64,07 & 5,86 & 68,06 \\
\hline \multirow{4}{*}{ Sudeste } & Minas Gerais & 87,59 & 4,46 & 91,69 & 97,90 & 0,83 & 98,73 & 43,72 & 16,72 & 52,50 & 68,20 & 4,78 & 71,62 \\
\hline & Espírito Santo & 86,18 & 4,95 & 90,67 & 97,80 & 0,84 & 98,64 & 40,00 & 19,11 & 49,46 & 67,39 & 5,00 & 70,94 \\
\hline & Rio de Janeiro & 93,53 & 2,01 & 95,45 & 99,26 & 0,24 & 99,50 & 53,19 & 11,47 & 60,08 & 70,27 & 4,54 & 73,61 \\
\hline & São Paulo & 93,86 & 2,00 & 95,78 & 99,17 & 0,29 & 99,46 & 54,80 & 11,47 & 61,90 & 70,90 & 4,35 & 74,13 \\
\hline \multirow{3}{*}{ Sul } & Paraná & 90,86 & 3,32 & 93,98 & 98,49 & 0,59 & 99,08 & 48,41 & 16,06 & 57,67 & 70,83 & 4,41 & 74,10 \\
\hline & Santa Catarina & 92,02 & 2,95 & 94,82 & 98,56 & 0,56 & 99,12 & 48,79 & 17,55 & 59,18 & 72,02 & 4,08 & 75,08 \\
\hline & Rio Grande do Sul & 92,22 & 2,69 & 94,78 & 98,81 & 0,44 & 99,26 & 49,77 & 15,03 & 58,58 & 70,89 & 4,24 & 74,04 \\
\hline \multirow{4}{*}{$\begin{array}{l}\text { Centro- } \\
\text { Oeste }\end{array}$} & Mato Grosso do Sul & 88,80 & 3,98 & 92,49 & 98,18 & 0,71 & 98,88 & 44,40 & 16,78 & 53,36 & 69,17 & 4,66 & 72,56 \\
\hline & Mato Grosso & 86,70 & 4,88 & 91,15 & 97,68 & 0,92 & 98,59 & 42,03 & 18,31 & 51,45 & 67,58 & 5,06 & 71,18 \\
\hline & Goiás & 90,17 & 3,32 & 93,27 & 98,60 & 0,52 & 99,11 & 46,42 & 14,69 & 54,42 & 68,73 & 4,50 & 71,97 \\
\hline & Distrito Federal & 93,13 & 2,29 & 95,32 & 99,05 & 0,34 & 99,39 & 53,47 & 13,14 & 61,57 & 70,00 & 4,62 & 73,39 \\
\hline Variação & Máximo & 93,86 & 14,11 & 95,78 & 99,26 & 2,90 & 99,50 & 54,80 & 38,16 & 61,90 & 72,02 & 7,13 & 75,08 \\
\hline Brasil & Mínimo & 65,53 & 2,00 & 76,30 & 92,21 & 0,24 & 94,94 & 19,63 & 11,47 & 31,46 & 60,01 & 4,08 & 64,63 \\
\hline
\end{tabular}

Fonte: elaboraçấo própria. 
tâncias exercem grande influência para o acesso ao saneamento, assegurando uma distribuição nada equitativa da baixa cobertura do serviço.

O acesso à educação básica revela características diversas das apresentadas pelo acesso à energia elétrica ou à água encanada, sendo melhor apenas se comparado ao saneamento. A desigualdade de oportunidade situa-se num intervalo sem muita dispersão, e o índice de oportunidade mostra que o país como um todo está longe de conquistar a universalização do acesso. A variaçáo da taxa de cobertura entre os estados se dá entre 64,63 e 75,08, o que demonstra que, dentro do território brasileiro, de $100 \%$ dos estudantes, $\mathrm{o}$ acesso a uma educação que lhe permita completar a sexta série com a idade correta ocorre somente para $64 \%$ a $75 \%$ dos indivíduos. Considerando que há que se descontar desse valor a penalidade em razão de a alocação da taxa de cobertura não ocorrer de forma equitativa, uma vez que há desigualdade de oportunidade, esse número reduz ainda mais, e o acesso justo e igualitário fica em torno de $60 \%$ a $72 \%$ dos casos. Entre as regiōes, os estados do Norte e do Nordeste se sobressaem com a desigualdade de oportunidade mais elevada associada com as menores taxas de cobertura do serviço, resultando, portanto, em menores $\mathrm{IOHs}$ para tais regióes. Acre e Maranhão despontam como estados com a desigualdade de oportunidade mais elevada e com os piores valores do IOH. A região Sul do país se destaca como a melhor no acesso a essa oportunidade: os três estados - Paraná, Santa Catarina e Rio Grande do Sul - possuem o maior acesso $(\mathrm{IOH})$ e a menor desigualdade de acesso.

Comparando-se os resultados aqui obtidos com o estudo realizado por Barros et al. (2009), o IOH para a eletricidade foi o que apresentou menor variação na relação feita entre os 19 países da amostra, sendo o intervalo de variaçáo de 42-98 para o ano de 1995 e de 45-99 para o de 2005. O Brasil obteve um IOH de 92 para essa oportunidade em 2005, de acordo com os autores. A dispersão maior observada foi para o saneamento, tal como ressaltado aqui. Esse serviço apresentou os piores indicadores: alta desigualdade de oportunidade e baixos Índices de Oportunidade Humana e taxa de cobertura. Ressaltando os resultados dos autores para o ano de 2005, na medida em que seu objetivo era analisar a evolução dos indicadores no período de 1995 a 2005, o IOH varia entre 11 e 87, e o índice de dissimilaridade, entre 4 e 49 , sendo esta a oportunidade com maior desigualdade no acesso para a maioria dos países. Para a completude da sexta série com idade correspondente 
e acesso à água, o IOH retorna valores de 24-86 e 41-97, respectivamente.

Vale mencionar que o objeto de pesquisa de Barros et al. (2009) envolve países onde há que se esperar que haja diferenças significativas entre os resultados no sentido de haver maior dispersáo entre os valores dos índices, principalmente pelo fato de analisarem tanto países emergentes quanto os considerados subdesenvolvidos. Quanto à análise aqui proposta para os estados brasileiros, tendo em vista que se trata de um mesmo território econômico e com um governo federal, esperar-se-ia que o desenvolvimento ocorresse para a naçáo como um todo. Entretanto, não é o que se verifica com base nos resultados encontrados. Uma vez que os serviços como água, energia elétrica e saneamento são considerados como infraestrutura básica, e a educação é meta primordial de nações em desenvolvimento, as diferenças entre os estados não deveriam ser tão elevadas quanto às observadas nos índices aqui reportados, com exceção da energia elétrica. As divergências entre os estados refletem políticas diferentes adotadas nas várias regióes, resultando em atrasos dentro de um mesmo país.

Os indicadores por oportunidade obtidos no presente estudo, em suma, revelam grandes disparidades entre os estados, tanto no que diz respeito à taxa de cobertura quanto à desigualdade de oportunidade. Há também diferenças no valor do IOH entre as oportunidades, sendo o saneamento o serviço básico que apresenta mais deficiências em todo o território brasileiro.

\section{4_Conclusões}

A literatura sobre a desigualdade de oportunidade, em contraste com a desigualdade de resultado, considera que a justiça social em uma sociedade está aliada à igualdade de oportunidade entre seus indivíduos. Tal conceito diverge da desigualdade de resultados, tema amplamente abordado por diversas pesquisas, uma vez que as diferenças observadas nos resultados, tais como renda ou nível de consumo, náo são consideradas socialmente injustas se as oportunidades são as mesmas para todos os indivíduos, independentemente de suas circunstâncias pessoais. Assim, se o acesso às oportunidades disponíveis apresenta correlaçáo com as características pessoais, origem familiar ou qualquer outra circunstância que o indivíduo não exerça controle direto, há desigualdade de oportunidade e, desse modo, injustiça social.

Tendo como foco a igualdade de oportunidade, este trabalho buscou mensurar um índice de oportunidade humana para o acesso a serviços básicos como 
energia elétrica, água limpa, saneamento e escola. Foram utilizados microdados da PNAD do ano de 2009 para indivíduos com idade até 16 anos para obter as probabilidades de acesso a partir da regressáo de um modelo logístico, sendo construído, então, o Índice de Oportunidade Humana que mensura as oportunidades disponíveis e alocadas de forma justa nos estados brasileiros.

Os resultados denotam grande disparidade no território brasileiro. $\mathrm{O}$ acesso à energia elétrica é o que apresenta maior taxa de cobertura e menor índice de desigualdade de oportunidade, sendo este o serviço com maior IOH dos quatro analisados. Já o saneamento se classifica como a oportunidade que possui o acesso mais precário por toda a população. $\mathrm{O}$ índice de dissimilaridade é elevado, e a taxa de cobertura, baixa, resultando num IOH baixo em todos os estados. $\mathrm{O}$ acesso à água limpa $\mathrm{e}$ o acesso à escola também apresentam diferenças entre as regióes, porém estas são mais discretas do que as observadas com relação ao saneamento, e os valores observados do índice para os estados também se apresentam melhores.

Os índices de oportunidade aqui mostrados apontam que a direçáo das políticas para a redução da desigualdade de oportunidade é a melhoria do acesso ao saneamento, serviço que apresenta a maior carência no país. As taxas de cobertura desse serviço devem ser estendidas e ampliadas. Isso não significa que as demais variáveis não precisam ser melhoradas; porém, o ponto mais crítico do país atualmente é o acesso ao saneamento. Com vistas a políticas regionais, Norte e Nordeste são as regiôes que apresentam a mais expressiva deficiência em todos os indicadores objetos da análise.

Uma extensão interessante do estudo da igualdade de oportunidade no acesso aos bens e serviços básicos seria a inclusão de uma variável que captasse a saúde como oportunidade na análise. Para que isso possa ser possível, entretanto, é necessária uma base de dados que contemple tal variável, o que hoje é fator limitante da PNAD. A construção do IOH para países desenvolvidos, bem como a posterior comparação com resultados aqui apresentados ou com os obtidos por Barros et al. (2009), é outra possível extensão. 


\section{Referências bibliográficas}

ARNESON, R. Equality of opportunity for welfare. Philosophical Studies, 56, p. 77 93, 1989.

BARROS, R. P. de; VEGA, J. M.; CHANDUVI, J. S. Measuring inequality of opportunities for children. Não publicado, World Bank, Washington, DC, 2008. Disponível em: <http:// siteresources.worldbank.org/ INTLACREGTOPPOVANA/ Resources/InenPaesdeBarros MolinasSaavedra.pdf $>$. Acesso em: 12/09/2010.

BARROS, R. P. de; FERREIRA, F. H. G; VEGA, J. M.; CHANDUVI, J. S. Measuring inequality of opportunities in Latin America and the Caribbean. Washington, DC: Palgrave Macmillan and the World Bank, 2009. 222 p.

BOURGUIGNON, François; FERREIRA, F. H. G.; MENÉNDEZ, $M$. Inequality of opportunity in Brazil. Review of Income and Wealth, Series, 53, n. 4, p. 585-618, Dez. 2007.

CHECCHI, D.; PERAGINE, V. Inequality of opportunity in Italy. Journal of Economic Inequality, 2009.
COHEN, G. A. On the currency of egalitarian justice. Ethics, 99, p. 906-944, 1989.

DWORKIN, R. What is equality? Part 1: Equality

of welfare; Part 2: Equality of resources. philos. Public Affairs, 10, p. 185-246; 283-345, 1981.

FIGUEIREDO, E.; ZIEGELMANN, F. Estimation of opportunity inequality in Brazil using nonparametric local logistic regression, Mimeo, 2010.

LEFRANC, A.; PISTOLESI, N.; TRANNOY, A. Inequality of opportunities vs. inequality of outcomes: Are western societies all alike? Review of Income and Wealth, 54, p. 513-546, 2008.

PERAGINE, V. Ranking of income distributions according to equality of opportunity. Journal of Income Inequality, 2 ,

p. 11-30, 2004.

RAMOS, X.; VAN DE GAER, D.

Empirical evidence on inequality of opportunity, 2009.

RAWLS, John. A Theory of

Justice. Harvard University Press, Cambridge, MA, 1971.
ROEMER, John E. Equality of opportunity. Harvard University Press, Cambridge, MA, 1998.

ROEMER, J. E. Theories of Distributive Justice.

Harvard University Press, Cambridge, MA, 1996.

VEGA, J. R. M.; BARROS, R. P. de; SAAVEDRA, J.; GIUGALE, M. Do our children have a chance? The 2010 Human Opportunity Report for Latin America and the Caribbean. World Bank, Washington, DC, 2010. 176 p.

WORLD BANK. World

Development Report 2006: Equity and development. Washington, DC: World Bank, 2006.

\section{YALONETZKY, Gaston.}

$A$ dissimilarity index of multidimensional inequality of opportunity. University of Oxford, Working paper no $39,2010$.

\begin{tabular}{|c|}
\hline E-mail de contato dos autores: \\
\hline flaviogonsalves@hotmail.com \\
\hline $\begin{array}{l}\text { Artigo recebido em abril de } 2011 \text { e } \\
\text { aprovado em setembro de } 2011\end{array}$ \\
\hline
\end{tabular}


Anexo

\section{Especificação das variáveis incluídas na análise}

\begin{tabular}{|c|c|c|}
\hline Variável & Denominação utilizada na equação (8) & Especificação \\
\hline Gênero & sexo & $\begin{array}{r}\text { Binária: } 1 \text { - masculino } \\
0-\text { feminino }\end{array}$ \\
\hline Raça & raça & $\begin{array}{r}\text { Binária: } 1 \text { - brancos } \\
0 \text { - náo brancos }\end{array}$ \\
\hline Área de residência & urbano & $\begin{array}{r}\text { Binária: } 1 \text { - urbana } \\
0-\text { rural }\end{array}$ \\
\hline Gênero da pessoa de referência & gen_pesref & $\begin{array}{r}\text { Binária: } 1-\text { masculino } \\
0-\text { feminino }\end{array}$ \\
\hline Presença da mãe & presença_mae & $\begin{array}{r}\text { Binária: } 1 \text { - está presente } \\
0 \text { - não está presente }\end{array}$ \\
\hline Educação da pessoa de referência do domicílio & educ_pesref & Quadrática \\
\hline Renda mensal domiciliar per capita & ln_renda & Logaritmo natural \\
\hline Número de pessoas no domicílio & num_pes & Linear \\
\hline
\end{tabular}

Fonte: elaboração própria.

\section{Correlação entre as variáveis}

\begin{tabular}{|c|c|c|c|c|c|c|c|c|}
\hline & sexо & raça & urbano & gen_pesref & presença_mae & educ_pesref & Inrenda & num_pes \\
\hline sexo & 1,000 & & & & & & & \\
\hline raça & $-0,012$ & 1,000 & & & & & & \\
\hline urbano & $-0,008$ & 0,078 & 1,000 & & & & & \\
\hline gen_pesref & 0,006 & 0,034 & $-0,161$ & 1,000 & & & & \\
\hline presenca_mae & $-0,002$ & 0,058 & 0,005 & 0,046 & 1,000 & & & \\
\hline educ_pesref & $-0,003$ & 0,182 & 0,212 & 0,010 & 0,135 & 1,000 & & \\
\hline lnrenda & 0,005 & 0,271 & 0,271 & 0,052 & $-0,016$ & 0,430 & 1,000 & \\
\hline num_pes & 0,002 & $-0,133$ & $-0,172$ & 0,131 & 0,060 & $-0,151$ & $-0,342$ & 1,000 \\
\hline
\end{tabular}

Fonte: elaboração própria. 